\title{
A POLARIZED PARTITION RELATION FOR CARDINALS OF COUNTABLE COFINALITY
}

\author{
ALBIN L. JONES
}

(Communicated by Julia Knight)

Abstract. We prove that if $\operatorname{cf} \kappa=\omega$ and $\lambda=2^{<\kappa}$, then

$$
\left(\begin{array}{c}
\lambda^{+} \\
\lambda
\end{array}\right) \rightarrow\left(\begin{array}{cc}
\lambda^{+} & \alpha \\
\lambda & \kappa
\end{array}\right)^{1,1}
$$

for all $\alpha<\omega_{1}$. This polarized partition relation holds if for every partition $\lambda \times \lambda^{+}=K_{0} \cup K_{1}$ either there are $B_{0} \in[\lambda]^{\lambda}$ and $A_{0} \in\left[\lambda^{+}\right]^{\lambda^{+}}$with $B_{0} \times A_{0} \subseteq$ $K_{0}$ or there are $B_{1} \in[\kappa]^{\lambda}$ and $A_{1} \in[\alpha]^{\lambda^{+}}$with $B_{1} \times A_{1} \subseteq K_{1}$.

\section{An introduction}

For ordinals $\alpha, \beta, \gamma_{0}, \gamma_{1}, \delta_{0}$, and $\delta_{1}$, the balanced polarized partition relation

$$
\left(\begin{array}{l}
\alpha \\
\beta
\end{array}\right) \rightarrow\left(\begin{array}{ll}
\gamma_{0} & \gamma_{1} \\
\delta_{0} & \delta_{1}
\end{array}\right)^{1,1}
$$

holds if for any partition of $\beta \times \alpha=K_{0} \cup K_{1}$ into two classes either there are $D_{0} \in[\beta]^{\delta_{0}}$ and $C_{0} \in[\alpha]^{\gamma_{0}}$ with $D_{0} \times C_{0} \subseteq K_{0}$ or there are $D_{1} \in[\beta]^{\delta_{1}}$ and $C_{1} \in[\alpha]^{\gamma_{1}}$ with $D_{1} \times C_{1} \subseteq K_{1}$. Similarly, for ordinals $\alpha, \beta, \gamma_{0}, \gamma_{1}, \delta_{0}, \delta_{1}$, and $\varepsilon$ the unbalanced polarized partition relation

$$
\left(\begin{array}{l}
\alpha \\
\beta
\end{array}\right) \rightarrow\left(\begin{array}{l}
\gamma_{0} \\
\delta_{0}
\end{array}\left(\begin{array}{l}
\gamma_{1} \\
\delta_{1}
\end{array}\right)_{\varepsilon}\right)^{1,1}
$$

holds if for any partition of $\beta \times \alpha=\bigcup_{\xi<1+\varepsilon} K_{\xi}$ into $1+\varepsilon$ classes either there are $D_{0} \in[\beta]^{\delta_{0}}$ and $C_{0} \in[\alpha]^{\gamma_{0}}$ with $D_{0} \times C_{0} \subseteq K_{0}$ or there are $\xi<\varepsilon, D_{1} \in[\beta]^{\delta_{1}}$, and $C_{1} \in[\alpha]^{\gamma_{1}}$ with $D_{1} \times C_{1} \subseteq K_{1+\xi}$. These relations are monotonic in the sense that they remain true if anything on the left side increases or anything on the right side decreases. They were first introduced and studied by P. Erdös and R. Rado in [4].

With A. Hajnal they proved in [3] that if one assumes the Generalized Continuum Hypothesis $(\mathrm{GCH})$, then the relation

$$
\left(\begin{array}{c}
\aleph_{\alpha+1} \\
\aleph_{\alpha}
\end{array}\right) \rightarrow\left(\begin{array}{cc}
\aleph_{\alpha+1} & \aleph_{0} \\
\aleph_{\alpha} & \aleph_{\alpha}
\end{array}\right)^{1,1}
$$

Received by the editors October 13, 2006 and, in revised form, February 15, 2007.

2000 Mathematics Subject Classification. Primary 03E05, 05D10; Secondary 05A18.

Key words and phrases. Transfinite cardinal, countable cofinality, elementary substructure, transfinite ordinal, polarized partition relation, Ramsey theory, regular cardinal, singular cardinal. 
holds for each infinite cardinal $\aleph_{\alpha}$ with cf $\aleph_{\alpha}=\aleph_{0}$. We remove the assumption of GCH and provide a direct proof that if $\operatorname{cf} \kappa=\omega$ and $\lambda=2^{<\kappa}$, then

$$
\left(\begin{array}{c}
\lambda^{+} \\
\lambda
\end{array}\right) \rightarrow\left(\begin{array}{cc}
\lambda^{+} & \alpha \\
\lambda & \kappa
\end{array}\right)^{1,1}
$$

for all $\alpha<\omega_{1}$.

\section{THE RESULT}

Suppose $\kappa$ and $\lambda$ are cardinals. A family $\left\{A_{\xi} \mid \xi \in \Theta\right\}$ is a $\kappa$-complete $\lambda$-uniform filter base or $(\kappa, \lambda)-f . b$. if $\left|\bigcap_{\xi \in X} A_{\xi}\right| \geq \lambda$ for all nonempty $X \in[\Theta]^{<\kappa}$. Note that any $(\kappa, \lambda)$-f.b. is also a $\left(\kappa_{0}, \lambda_{0}\right)$-f.b. for all $\kappa_{0} \leq \kappa$ and $\lambda_{0} \leq \lambda$.

Lemma 1. Suppose $\kappa$ and $\lambda$ are infinite cardinals with $\lambda$ regular and $\kappa<\lambda$. For $A \subseteq \kappa$, let $\bar{A}=\kappa \backslash A$. Then for any $\left\{A_{\xi} \mid \xi<\lambda\right\} \subseteq \mathcal{P}(\kappa)$ either

(a) there is $X \in[\lambda]^{\lambda}$ such that $\left\{A_{\xi} \mid \xi \in X\right\}$ is an $(\omega, \kappa)$-f.b., or

(b) there is $Y \in[\lambda]^{\lambda}$ such that $\left|\bigcap_{\xi \in Y} \overline{A_{\xi}}\right|=\kappa$.

Proof. For $x \in[\lambda]^{<\omega}$, let $A(x)=\bigcap_{\xi \in x} A_{\xi}$. Fix $X \subseteq \lambda$ maximal such that $\left\{A_{\xi} \mid \xi \in\right.$ $X\}$ is an $(\omega, \kappa)$-f.b. Suppose that $|X|<\lambda$. Let $\mu=\max \{\kappa,|X|\}$. Fix $M \prec H_{\lambda^{+}}$ with $|M|=\mu, \mu \cup\{\mu\} \cup X \cup\{X\} \subseteq M$, and $\left\{A_{\xi} \mid \xi<\lambda\right\} \in M$. Let $\theta=\sup (\lambda \cap M)$. Choose $\delta \in \lambda \backslash \theta$ arbitrarily. By the maximality of $X$, there must be $x \in[X]^{<\omega}$ with $\left|A_{\delta} \cap A(x)\right|<\kappa$. Note that $A(x) \in M$ so there must be in $M$ a pairwise disjoint collection $\left\{B_{\eta} \mid \eta<\kappa\right\} \subseteq[A(x)]^{\kappa}$ with $A(x)=\bigcup_{\eta<\kappa} B_{\eta}$. Because $\left|A_{\delta} \cap A(x)\right|<\kappa$, there must be $\eta<\kappa$ with $A_{\delta} \cap B_{\eta}=\emptyset$. Let $Y=\left\{\xi<\lambda \mid A_{\xi} \cap B_{\eta}=\emptyset\right\}$. Then $B_{\eta} \in[\kappa]^{\kappa}$ by construction, $Y \in[\lambda]^{\lambda}$ by elementarity, and $B_{\eta} \subseteq \bigcap_{\xi \in Y} \overline{A_{\xi}}$ by definition. Thus $\left|\bigcap_{\xi \in Y} \overline{A_{\xi}}\right|=\kappa$.

Corollary 2 (P. Erdős, A. Hajnal, and R. Rado for $\left.\lambda=\kappa^{+}\right)$. The relation

$$
\left(\begin{array}{l}
\lambda \\
\kappa
\end{array}\right) \rightarrow\left(\begin{array}{ll}
\lambda & n \\
\kappa & \kappa
\end{array}\right)^{1,1}
$$

holds whenever $n<\omega \leq \kappa<\lambda$ and $\lambda$ is regular.

Note that in part (b) of the lemma we could require the set $Y$ to be stationary (by taking $\delta=\theta$ ), to be contained in any particular element of $[\lambda]^{\lambda} \cap M$ (by taking $\delta$ from that set and adding that property to the definition of $Y$ ), or perhaps both. We could, for example, require that $Y$ be stationary in $\lambda$ and consist only of ordinals of countable cofinality. This could be accomplished by choosing $M$ with $\operatorname{cf} \theta=\omega$, taking $\delta=\theta$, and adding " $\mathrm{cf} \xi=\omega$ " to the definition of $Y$.

Lemma 3. If $\lambda=2^{<\kappa}$ and $\left\{A_{\xi} \mid \xi<\lambda^{+}\right\} \subseteq[\lambda]^{\lambda}$ is a (cf $\left.\kappa, \kappa\right)$-f.b., then for every $\alpha<(\mathrm{cf} \kappa)^{+}$there is $X \in\left[\lambda^{+}\right]^{\alpha}$ with $\left|\bigcap_{\xi \in X} A_{\xi}\right| \geq \kappa$.

Proof. Fix a sequence of cardinals $\left\{\kappa_{\iota} \mid \iota<\operatorname{cf} \kappa\right\} \subseteq \kappa$ with

$$
\kappa=\sum_{\iota<\mathrm{cf} \kappa} \kappa_{\iota} \text { and } \quad \text { ef } \kappa \notin\left\{\operatorname{cf} \kappa_{\iota} \mid \iota<\operatorname{cf} \kappa\right\} .
$$

Choose a regular cardinal $\theta$ greater than $\lambda^{+}$. Construct a sequence $\left\{M_{\eta} \mid \eta \leq \alpha\right\}$ of elementary substructures of $H_{\theta}$ such that $\left|M_{\eta}\right|=\lambda, \lambda+1 \subseteq M_{\eta},\left[M_{\eta}\right]<\mathrm{cf} \kappa \subseteq M_{\eta}$, and $\left\{A_{\xi} \mid \xi<\lambda^{+}\right\}, M_{\zeta} \in M_{\eta}$ whenever $\zeta<\eta \leq \alpha$. Choose $\delta \in \lambda^{+} \backslash M_{\alpha}$ arbitrarily. For $x \in\left[\lambda^{+}\right]^{<\operatorname{cf} \kappa}$, let $A(x)=\bigcap_{\xi \in x} A_{\xi}$. For $a \in[\lambda]^{<\kappa}$, let $X(a)=\left\{\xi<\lambda^{+} \mid a \subseteq A_{\xi}\right\}$. 
Claim A. Suppose that $\iota<\mathrm{cf} \kappa$. Then for any $x \in[\lambda]^{\kappa_{\iota}}$ there is $y \in[\lambda]^{\kappa_{\iota}} \cap M_{0}$ with $y \subseteq x$. Consequently, $[A]^{\kappa_{\iota}} \cap M_{0}$ is nonempty for all $A \subseteq \lambda$ with $|A| \geq \kappa$.

Proof. Because $|x|=\kappa_{\iota}$ and $\operatorname{cf} \kappa_{\iota} \neq \operatorname{cf} \kappa$ there must be $v<\kappa$ with $\left|x \cap 2^{\kappa_{v}}\right|=\kappa_{\iota}$. Note that $\left[2^{\kappa_{v}}\right]^{\kappa_{\iota}} \subseteq M_{0}$ because $\left[2^{\kappa_{v}}\right]^{\kappa_{\iota}} \in M_{0}$ and $\left|\left[2^{\kappa_{v}}\right]^{\kappa_{\iota}}\right|=\left(2^{\kappa_{v}}\right)^{\kappa_{\iota}} \leq 2^{<\kappa}=\lambda$. Let $y=x \cap 2^{\kappa_{v}}$. Then $y \in[\lambda]^{\kappa_{\iota}} \cap M_{0}$ and $y \subseteq x$.

Claim B. Suppose that $\iota<\mathrm{cf} \kappa$ and $\eta<\alpha$. Then for any $a \in\left[A_{\delta}\right]^{<\kappa} \cap M_{0}$, $x \in[X(a)]^{<\mathrm{cf} \kappa} \cap M_{\alpha}$, there are $\bar{a} \in\left[A_{\delta}\right]^{<\kappa} \cap M_{0}$ and $\bar{x} \in[X(\bar{a})]^{<\mathrm{cf} \kappa} \cap M_{\alpha}$ with $\bar{a} \supseteq a, \bar{x} \supseteq x,|\bar{a} \backslash a| \geq \kappa_{\iota}$, and $\bar{x} \cap M_{\eta+1} \backslash M_{\eta} \neq \emptyset$.

Proof. Let $a \in\left[A_{\delta}\right]^{<\kappa} \cap M_{0}$ and $x \in[X(a)]^{<\kappa} \cap M_{\alpha}$ be given. Let $A=A(x) \cap A_{\delta}$. Note that $|A| \geq \kappa$, since $\left\{A_{\xi} \mid \xi<\lambda^{+}\right\}$is a $(\operatorname{cf} \kappa, \kappa)$-f.b. By Claim A, $[A \backslash a]^{\kappa_{\iota}} \cap M_{0}$ is nonempty. Choose $b \in[A \backslash a]^{\kappa_{\iota}} \cap M_{0}$ arbitrarily and let $\bar{a}=a \cup b$. Let $X=$ $X(\bar{a}) \backslash M_{\eta}$. Note that $X$ is in $M_{\eta+1}$ (since it is definable in $M_{\eta+1}$ ) and nonempty (since it includes $\delta$ ). By elementarity, $X \cap M_{\eta+1}$ must be nonempty. Choose $\xi \in X \cap M_{\eta+1}$ arbitrarily, and let $\bar{x}=x \cup\{\xi\}$.

Let $\{\xi(\iota) \mid \iota<$ cf $\kappa\}$ be an enumeration of (the ordinals less than) $\alpha$ in order type cf $\kappa$ (with repetitions, perhaps). Repeated use of Claim B (taking unions at the limits) allows us to construct sequences of sets

$$
\emptyset=a_{0} \subseteq a_{1} \subseteq a_{2} \subseteq \cdots \subseteq a_{\iota} \subseteq \cdots \subseteq A_{\delta}
$$

and

$$
\emptyset=x_{0} \subseteq x_{1} \subseteq x_{2} \subseteq \cdots \subseteq x_{\iota} \subseteq \cdots \subseteq \lambda^{+} \cap M_{\alpha}
$$

such that $a_{\iota} \subseteq A_{\delta}, x_{\iota} \subseteq X\left(a_{\iota}\right)$, ot $\left(a_{\iota}\right) \geq \kappa_{\iota}$, and $x_{\iota} \cap M_{\xi(\iota)+1} \backslash M_{\xi(\iota)} \neq \emptyset$ for each $\iota<\kappa$. Let $A=\bigcup_{\iota<\operatorname{cf} \kappa} a_{\iota}$ and $X=\bigcup_{\iota<\operatorname{cf} \kappa} x_{\iota}$. Then ot $(A) \geq \kappa$, ot $(X) \geq \alpha$, and $A \subseteq \bigcap_{\xi \in X} A_{\xi}$.

Among other things, this lemma immediately implies that if $\kappa^{<\kappa}=\kappa$ and there is a $\kappa$-dense ideal on $\kappa$, then

$$
\left(\begin{array}{c}
\kappa^{+} \\
\kappa
\end{array}\right) \rightarrow\left(\begin{array}{l}
\alpha \\
\kappa
\end{array}\right)^{1,1}
$$

for all $\alpha<\kappa^{+}$. This is true for $\kappa=\omega$ or for $\kappa$ measurable and uncountable, for example. These last two relations were first proven by Baumgartner and Hajnal in [1] and Čudnovskiı̌ in [2], respectively.

Proposition 4. If $\mathrm{cf} \kappa=\omega$ and $\lambda=2^{<\kappa}$, then

$$
\left(\begin{array}{c}
\lambda^{+} \\
\lambda
\end{array}\right) \rightarrow\left(\begin{array}{cc}
\lambda^{+} & \alpha \\
\lambda & \kappa
\end{array}\right)^{1,1}
$$

for all $\alpha<\omega_{1}$. Consequently,

$$
\left(\begin{array}{c}
\lambda^{+} \\
\lambda
\end{array}\right) \rightarrow\left(\begin{array}{c}
\lambda^{+} \\
\lambda
\end{array}\left(\begin{array}{l}
\alpha \\
\kappa
\end{array}\right)_{m}\right)^{1,1}
$$

for all $\alpha<\omega_{1}$ and $m<\omega$.

Proof. Suppose $\lambda \times \lambda^{+}=K_{0} \cup K_{1}$. For $\xi<\lambda^{+}$and $i \in\{0,1\}$ put

$$
K_{i}^{\xi}=\left\{\eta<\lambda \mid(\eta, \xi) \in K_{i}\right\} .
$$

By Lemma 1 either (a) there is $X \in\left[\lambda^{+}\right]^{\lambda^{+}}$such that $\left\{K_{1}^{\xi} \mid \xi \in X\right\}$ is an $(\omega, \lambda)$ f.b., or (b) there is $Y \in\left[\lambda^{+}\right]^{\lambda^{+}}$such that $\left|\bigcap_{\xi \in Y} K_{0}^{\xi}\right|=\lambda$. If the former, then by 
Lemma 3 there are $A_{1} \in\left[\lambda^{+}\right]^{\alpha}$ and $B_{1} \in[\lambda]^{\lambda}$ with $B_{1} \times A_{1} \subseteq K_{1}$. If the latter, then clearly there are $A_{0} \in\left[\lambda^{+}\right]^{\lambda^{+}}$and $B_{0} \in[\lambda]^{\lambda}$ with $B_{0} \times A_{0} \subseteq K_{0}$.

The comments following Lemma 1 yield the following.

Corollary 5. If $\operatorname{cf} \kappa=\omega, \lambda=2^{<\kappa}$, and $\mu \leq \lambda$, then

$$
\left(\begin{array}{c}
\text { stat } \lambda^{+} \\
\lambda
\end{array}\right) \rightarrow\left(\begin{array}{c}
\text { stat } \lambda^{+} \\
\lambda
\end{array}\left(\begin{array}{l}
\alpha \\
\kappa
\end{array}\right)_{m}\right)^{1,1}
$$

for all $\alpha<\omega_{1}$ and all $m<\omega$. In other words, for any stationary $S \subseteq \lambda^{+}$and partition $\lambda \times S=\bigcup_{i<1+m} K_{i}$, either there are $B_{0} \in[\lambda]^{\lambda}$ and stationary $S_{0} \subseteq S$ with $B_{0} \times S_{0} \subseteq K_{0}$ or there are $i<m, B_{1} \in[\lambda]^{\kappa}$, and $A_{1} \in[S]^{\alpha}$ with $B_{1} \times A_{1} \subseteq K_{1+i}$.

\section{A CONCLUSION}

These results are almost optimal in the sense that in [3] it is proven from GCH that if $\mathrm{cf} \aleph_{\alpha}>\aleph_{0}$, then

$$
\left(\begin{array}{c}
\aleph_{\alpha+1} \\
\aleph_{\alpha}
\end{array}\right) \nrightarrow\left(\begin{array}{cc}
\aleph_{\alpha+1} & \aleph_{0} \\
\aleph_{\alpha} & \aleph_{\alpha}
\end{array}\right)^{1,1}
$$

and if $\operatorname{cf} \aleph_{\alpha}=\aleph_{0}$, then

$$
\left(\begin{array}{c}
\aleph_{\alpha+1} \\
\aleph_{\alpha}
\end{array}\right) \nrightarrow\left(\begin{array}{cc}
\aleph_{\alpha+1} & \aleph_{2} \\
\aleph_{\alpha} & \aleph_{\alpha}^{1,1}
\end{array}\right)^{1}
$$

To our knowledge, however, the following question of [3] remains unanswered.

Question 1. Does GCH imply that

$$
\left(\begin{array}{c}
\aleph_{\omega+1} \\
\aleph_{\omega}
\end{array}\right) \rightarrow\left(\begin{array}{cc}
\aleph_{\omega+1} & \aleph_{1} \\
\aleph_{\omega} & \aleph_{\omega}
\end{array}\right)^{1,1} ?
$$

The negative resolution of the next two questions would considerably sharpen the results presented above.

Question 2. Is it consistent that

$$
\left(\begin{array}{c}
\left(2^{<\kappa}\right)^{+} \\
2^{<\kappa}
\end{array}\right) \rightarrow\left(\begin{array}{cc}
\left(2^{<\kappa}\right)^{+} & \omega_{1} \\
2^{<\kappa} & \kappa
\end{array}\right)^{1,1}
$$

for all $\kappa$ with cf $\kappa=\omega$ ?

Question 3. Is it possible that there is $\kappa$ with $\mathrm{cf} \kappa>\omega$ for which

$$
\left(\begin{array}{c}
\left(2^{<\kappa}\right)^{+} \\
2^{<\kappa}
\end{array}\right) \rightarrow\left(\begin{array}{cc}
\left(2^{<\kappa}\right)^{+} & \omega \\
2^{<\kappa} & \kappa
\end{array}\right)^{1,1} ?
$$

And finally, a related question of independent interest:

Question 4. Is it consistent that there is $\kappa>\omega$ for which

$$
\left(\begin{array}{c}
\kappa^{+} \\
\kappa
\end{array}\right) \rightarrow\left(\begin{array}{cc}
\kappa^{+} & \kappa \\
\kappa & \kappa
\end{array}\right)^{1,1} ?
$$




\section{REFERENCES}

1. J. Baumgartner and A. Hajnal, A proof (involving Martin's axiom) of a partition relation, Fund. Math. 78 (1973), no. 3, 193-203. MR0319768 (47:8310)

2. G. V. Čudnovskiı̌, Combinatorial properties of compact cardinals, Infinite and finite sets (Colloq., Keszthely, 1973; dedicated to P. Erdős on his 60th birthday), Vol. I, North-Holland, Amsterdam, 1975, pp. 289-306. Colloq. Math. Soc. János Bolyai, Vol. 10. MR0371655 (51:7873)

3. P. Erdős, A. Hajnal, and R. Rado, Partition relations for cardinal numbers, Acta Math. Acad. Sci. Hungar. 16 (1965), 93-196.

4. P. Erdős and R. Rado, A partition calculus in set theory, Bull. Amer. Math. Soc. 62 (1956), 427-489. MR0081864 (18:458a)

2153 OAkdale Rd., Pasadena, Maryland 21122

E-mail address: alj@mojumi.net 